findings. Between ages 9 months and 2 years, MRIs showed signal abnormalities of deep cerebral white matter, corpus callosum, thalamus, basal ganglia, brainstem, and cerebellar white matter. A rim of periventricular white matter was preserved. At follow-up, abnormalities gradually improved. Clinical manifestations were correlated with the MRI findings. Clinical regression occurred in the second half-year of life with limb spasticity, axial hypotonia, and loss of milestones and accompanied by elevated blood and CSF lactate levels. After 2 years of age, patients showed clinical improvement and normal lactate. Lysosomal enzyme activities in leukocytes, especially arylsulfatase A and galactocerebrosidase, were normal. Acylcarnitine, amino acid, urinary organic acid, and very-long-chain fatty acid levels were normal. Mitochondrial DNA screening for mutations was negative. The patients represented a single novel leukoencephalopathy, probably caused by a mitochondrial defect. (Steenweg ME, Vanderver A, Ceulemans B, et al. Novel infantile-onset leukoencephalopathy with high lactate level and slow improvement. Arch Neurol 2012 June;69(6):718-722). (Respond: Marjo S van der Knaap MDPhD, Department of Child Neurology, VU University Medical Center, De Boelelaan 1117, 1081 HV Amsterdam, the Netherlands (E-mail: ms.vanderknaap@vumc.nl).

COMMENT. Metachromatic leukodystrophy, Krabbe's disease, Alexander disease, adrenoleukodystrophy, and known mitochondrial diseases were excluded.

The first author of the above paper also reports a leukoencephalopthy with thalamus and brainstem involvement and high lactate 'LTBL' caused by EARS2 mutations. (Steenweg ME et al. Brain 2012 May;135(Pt5):1387-1394). In a cohort of 12 patients, this mitochondrial encephalopathy was either mild or severe, with infantile onset, rapidly progressive MRI abnormalities and increased lactate. Patients in the mild group partially recovered and regained milestones at follow-up, MRI improved, and lactate levels fell toward normal. Patients in the severe group had persistent neurological regression, brain atrophy on MRI, and increased lactate. MRI was hallmarked by symmetrical cerebral white matter abnormalities sparing the periventricular rim, and symmetrical abnormalities of the thalami, brain stem, and cerebellar white matter.

\title{
INFANTILE-ONSET ALEXANDER DISEASE
}

Researchers at the All India Institute of Medical Sciences, New Delhi, India, and VU University Medical Center, Amsterdam report a 6-year-old Indian boy with infantileonset Alexander disease having an unusually mild clinical course and a de novo mutation in the glial fibrillary acidic protein (GFAP) gene. He had a large head and delayed motor milestones since infancy. He sat at 2 years of age and walked at 3 and $1 / 2$ years. Left focal seizures at 3 years of age were controlled by sodium valproate. Neuro exam revealed hypotonia, brisk reflexes, and extensor plantar responses. MRI showed T2 signal changes in cerebral white matter, mainly frontal. Genetic analysis confirmed the diagnosis and his condition had remained stable for 18 months. (Ramesh K, Sharma S, Kumar A, Salomons GS, van der Knaap MS, Gulati S. Infantile-onset Alexander disease: A genetically proven case with mild clinical course in a 6-year-old Indian boy. J Child Neurol 2012 May 7 [Epub ahead of print]). (Response: Sheffali Gulati MD. E-mail: sheffaligulati@gmail.com). 